\title{
Lecture de Daniel dans la Fosse aux Lions
}

Reading of Daniel in the Lion's Den

\section{Michael Palmer}

\section{OpenEdition}

Journals

Édition électronique

URL : http://journals.openedition.org/questionsdecommunication/7703

DOI : 10.4000/questionsdecommunication. 7703

ISSN : 2259-8901

\section{Éditeur}

Presses universitaires de Lorraine

\section{Édition imprimée}

Date de publication : 1 décembre 2006

Pagination : 225-233

ISBN : $978-2-86480-828-2$

ISSN : 1633-5961

\section{Référence électronique}

Michael Palmer, «Lecture de Daniel dans la Fosse aux Lions », Questions de communication [En ligne], 10 | 2006, mis en ligne le 01 décembre 2006, consulté le 22 mars 2021. URL : http://

journals.openedition.org/questionsdecommunication/7703; DOI : https://doi.org/10.4000/ questionsdecommunication.7703 


\section{$>$ ÉCHANGES}

MICHAEL PALMER

Centre d'histoire des récits de l'information et des médias en Europe

Université Paris 3

Michael.Palmer@univ-paris3.fr

\section{LECTURE DE DANIEL DANS LA FOSSE AUX LIONS}

Résumé. - L'acuité du regard de Daniel Dayan conduit à penser autrement la couverture de l'actualité internationale par les médias. Un texte se lit en contexte. Ici, on lit, ou relit, la contribution de Daniel Dayan (Questions de communication, 8, 2005) pendant que l'on relit des textes anciens sur la couverture du conflit israélopalestinien et les questions liées à l'Islam. On le fait aussi pendant que les médias internationaux couvrent la confrontation entre Israël et le Hezbollah, au cours l'été 2006. Ce qui n'est pas sans conséquence sur le propos.

Mots clés. - Seconde Intifada, guerre au Liban, mots, images, dire la guerre, agences de presse, internet. 
'entretien de Daniel Dayan (2005) avec Béatrice Fleury et Jacques Walter comporte deux intertitres: "Les médias français et Israël », «Questions de méthode ». Je rebondirai sur plusieurs des points soulevés dans chacun, mais en partant de deux remarques générales : « La plupart des chercheurs français sont passés à coté de thèmes qui $[\ldots]$ auraient pu les mobiliser. Ils ont laissé le champ libre à d'autres : à des militants, à des journalistes » (ibid. : 219) et « II est absurde de se livrer à une interpellation destinée à son seul disque dur » (ibid. : 197).

\section{La recherche sur les médias et la « babélisation » du savoir}

Je sais mal ce que serait la recherche française, comparée à l'américaine ou à la britannique. En revanche, l'individu devant son « ordi », celui-là, je le connais. Quiconque accède aux portails, pratique les blogs, consulte les sites web n'ignore pas que l'information ainsi obtenue et l'adrénaline produite lors des « échanges » réalisés, s'accompagnent d'une fragmentation et d'une « babélisation » du savoir, d'une vulgarisation des discours et, parfois, d'une confusion des esprits. Certes, au cours de la moitié et de la fin du XIXe siècle, on en disait autant de l'impact de l'information télégraphique, tel parmi d'autres, Émile Zola qui s'inquiétait de l'influence, au réveil, de la nervosité occasionnée par l'accumulation des dernières dépêches télégraphiques de la nuit sur les esprits... Souvent, «l'info-communicant » est dans la position de l'arroseur arrosé. Plus il cerne son objet, peaufine ses méthodes, plus il sait que son essence même - «l'info-com » - est un syntagme incontournable qui, en tension constante, le renvoie toujours à ce point de départ suspendu au carrefour d'où rayonnent d'innombrables chemins de traverse. Hermès, dieu tutélaire toujours. Analogies, métaphores et métonymies sont aussi constitutives de l'interdiscipline que l'est l'étude des théories et des pratiques.

Daniel Dayan est lui-même habité par Sigmund Freud et Erving Goffman ou par James W. Carey et Elihu Katz. Aussi s'intéresse-t-il aux ratés discursifs - donc révélateurs, mais de quoi ?... - comme par la prégnance du visuel, chez le récepteur-spectateur tout au moins. Finalement, la pathologie et le symbolisme importeraient plus que les «factualités 》(Dayan, 2005 : 217) - factualités qui pourraient s'avérer « parallèles », n'en déplaise au postulat d'un débat sur des bases partageables d'un monde commun - d'où la possibilité d'un discours sur l'objectivité. On salue, mais s'étonne presque, lorsque Daniel Dayan (ibid. : 219) propose une taxinomie de catégories permettant l'étude quantitative du traitement du Proche-Orient par les médias français 
pendant la seconde Intifada: "Les normes du discours de l'information », c'est l'Arlésienne, tout comme la quête de l'objectivité ce qui ne signifie pas qu'il ne faille pas méditer sur les efforts de ceux qui les recherchent.

Le scientifique est hybride, tant par sa posture que par sa culture. Face à l'information-news - produit lui aussi hybride, tant par la complexité de sa production, son traitement, sa transmission et sa réception -, le chercheur négocie les prismes de la représentation. "L'actualité », pensée en contexte et avec une glose parfois stéréotypée - IsraëlPalestine/Juifs-Arabes/Occupation-Résistance... -, en témoigne. Les binômes qui scandent la vie d'un chercheur-citoyen méditerranéen et transatlantique, pratiquant à la fois des zooms pertinents - la facticité du terme Proche-Orient (Dayan, 2005 : 200-20l), la double (au moins) lecture de deux événements survenus la même semaine (ibid. : 213), ce Jérusalem historique qui se tiendrait à l'intérieur de la place de la Concorde (ibid. :215) - et des visions surplombantes - s'il n'y a plus de « grands récits, $[\ldots]$ que se passe-t-il quand il ne se passe rien ? 》 (ibid. : 212) - hantent les questions que se pose le citoyen-chercheur nomade.

Existerait-il - non pas chez Daniel Dayan, mais dans la communauté de ceux qui veulent trouver du sens aux discours que le monde tient sur le monde - comme une nostalgie des « Grands Récits 》? Jadis, la mise en parallèle du « sionisme et du racisme sud-africain » (ibid. : 21 I) faisait partie de ces Récits. De même en était-il de l'opposition Est-Ouest (« républiques socialistes populaires » face au « monde libre »), ou des récits gréco-judéo-chrétien-musulman..., du pourtour méditerranéen et du « croissant fertile »... Pour Daniel Dayan (ibid. : 206) - et il n'a pas tort - «à partir du moment où la nature de l'information est prédéterminée par l'existence d'un récit, c'est l'ensemble des informations offertes qui se trouve frappé de discrédit ». Est-ce donc par l'attention flottante des psychanalystes (ibid. : 207) que l'on peut capter les « gestes » visuels ou textuels qui contredisent la monstration ? La tension visuelle-textuelle - avec l'oral en fée du logis - hante la production média, avec l'apparence (mais seulement ?) d'une prégnance croissante du visuel : « show, not tell » [montrez, ne dites pas], répète-ton à satiété dans les agences d'information. Quant à cette info-news ellemême, qu'il s'agisse du proche et du lointain, du pic événementiel et de la latence, du hard/spot et du soft, du grave et de l'insolite..., il arrive que la nomenclature du chercheur rejoigne les distinctions opérées par le journaliste lui-même. Mais leurs regards, certes, diffèrent. Le second est aux prises avec les circonstances de l'immédiat, résumées par ce néologisme des 30 dernières années - «breaking news »-, tout en cherchant une forme de distanciation pour « mettre en contexte » (ce qui n'est pas la même chose que commenter/éditorialiser). Quant au 
chercheur, il a un autre agenda. Avec courage, Daniel Dayan revisite ici, ainsi que dans son ouvrage Le théâtre de la terreur.Terrorisme et télévision (2006), les deux ans de couverture médiatique française après le début de la seconde Intifada pour savoir si celle-ci permettait une meilleure compréhension « de ce qui se passe au Proche-Orient ». Et à travers cette analyse, il se donne aussi pour mission d'« intervenir »-pour employer un terme fétiche de Pierre Bourdieu. Or, de telles interventions-interpellations sont très pratiquées par certains enseignants-chercheurs aux États-Unis et le seraient éventuellement davantage aujourd'hui par leurs collègues français que par le passé. À moins que ce ne soit plutôt que, par le passé, la manière d'agir de nos devanciers se manifestait différemment?

Finalement, lire Daniel Dayan, c'est se remémorer d'autres travaux. En commençant par ceux d'Edward Said, Covering Islam : How the media and the experts determine how we see the rest of the world (1981), et en poursuivant par deux textes récents, celui de Joss Dray et Denis Sieffert, La guerre israélienne de l'information. Désinformation et fausses symétries dans le conflit israélo-palestinien (2002), et celui de Greg Philo et Mike Berry, Bad news from Israel (2004). Tout en ayant leurs propres caractéristiques, ces textes se situent dans la mouvance des travaux de Noam Chomsky et Edward S. Herman, dont Manufacturing consent. The Political Economy of the Mass Media. Une formule [Les Forgerons du consensus] - qui, elle-même, reprend une phrase de Walter Lippmann. Pour nous, géopolitique, géo-économie et géo-information - et, à terme, médiatisation - sont à penser ensemble. Les visions du monde se croisent, s'interconnectent et connaissent des ratés. Daniel Dayan attire l'attention sur les outils qui permettent de les appréhender. Ici, je voudrais moins questionner les ratés discursifs qu'il pointe - en identifiant des écarts entre le montré et le dit ou entre l'image et le texte d'accompagnement - qu'évoquer in fine l'adjuvant qu'apporte - pour employer une formule à la Régis Debray - la blogosphère, l'en-ligne, la vidéosphère à la graphosphère - côté information-news s'entend.

\section{Des consignes professionnelles à leurs usages}

« C'est ici un fait, pas un jugement de valeur ». De telles phrases scandent les consignes rédactionnelles, telles des évaluations de la copie, formulées au sein de ces multinationales, plurilingues, multimédias que sont les agences d'information comme Reuters, AP (qui ont chacune une « division » agences d'images), et l'AFP (dont la production d'images photo, infographie et vidéo - augmente). Le manuel de l'AFP (2004) ne renvoie pas expressément au vocabulaire convoqué lors de la seconde Intifada. II rappelle une note rédactionnelle du 22 octobre 2002, fruit d'une réflexion associant la rédaction en chef pour le Proche-Orient, le 
bureau de Jérusalem et la rédaction en chef centrale.Y sont précisées les conditions d'emploi de termes tels que « colon », 《implantation », 《 terrorisme », « extrémiste » ou « assassinat ». Par exemple, « assassinat » ne peut s'employer « que dans le cas où il s'agit d'un meurtre avec préméditation avéré, incontestable et reconnu comme tel par les autorités ou l'armée israélienne... Dans tous les autres cas, lorsqu'il s'agit d'une opération ciblée d'élimination, nous devons utiliser le terme "éliminer" avec, dans la copie, au moins un paragraphe pour établir le fait que l'armée israélienne a déclenché une opération ciblée concernant une personne déterminée. En anglais, le terme utilisable pour "assassiné" reste "killed" ».

Reste à savoir si de telles consignes sont toujours respectées, et si les clients et usagers de la copie d'agence que sont les médias emploient de telles précautions. Certes, il arrive que les transnationales de l'information rappellent que des dirigeants arabes - Yasser Arafat, Ben Laden acceptent eux-mêmes le terme «terroriste 》: « Le président de l'Autorité palestinienne Yasser Arafat a lui-même utilisé le qualificatif de terroriste pour les attentats et attaques menés contre des populations civiles à l'intérieur d'Israël » (AFP, 2004 ; Palmer, 2006). De même, au premier journaliste travaillant pour des médias internationaux - AlJazira, en l'occurrence - auquel Ben Laden accorda un interview après les attentats du I I-Septembre, ce dernier répond : « Si inciter... c'est du terrorisme, si tuer ceux qui tuent nos fils c'est du terrorisme, et bien que l'Histoire atteste que nous sommes des terroristes ». Les médias internationaux questionnent-ils autant le vocabulaire grâce auquel les autorités israéliennes qualifient les faits et gestes de Tsahal, l'armée d'Israël ? Poser la question, est-ce témoigner d'un parti-pris ? Évoquer le passé controversé, polysémique de l'emploi de vocables à problème tel « terroriste », est-ce se contenter d'une fuite en avant... vers le passé ? Lorsque, dans les années 40, les autorités britanniques administraient cette Palestine qui allait devenir Israël en 1948, elles qualifiaient les actes violents du groupe Stern, de Menahem Begin... de « terroristes ».

\section{De la seconde Intifada à la guerre du Liban}

Toujours est-il que les remarques de Daniel Dayan avivent les plaies de mes « échanges » avec le disque dur précité. J'écris ces lignes en juillet 2006, peu après le début de l'offensive d'Israël contre le Hezbollah au sud-Liban et à Gaza. Daniel Dayan m'amène à me replonger dans un corpus d'évaluations quasi quotidiennes de la copie Reuters (en anglais) produite entre 1998 et 2002, évaluations réalisées à New York et à Washington. Le 19 novembre 200I, un observateur écrivait: «Attention : il y a quelques semaines, il semblait que dans chaque papier concernant le Moyen-Orient, on écrivait "les État-Unis, allié et gardien 
d'Israël" : "Israel's guardian ally" - ne pas trop en faire » (« Les ÉtatsUnis, allié et soutien d'Israël » figure dans l'article à la Une du Monde du 28 juillet 2006). À la mi-juillet 2006, l'actualité me fait scruter la copie produite dans l'édition internationale du Times de Londres, I'hebdomadaire anglais-américain international The Economist, et le quotidien International Herald Tribune $(I H T)$ - édition internationale/ mondiale, rédigée à Paris et à Hong Kong du New York Times. Pointons quelques «trouvailles ». La légende d'une photo sourcée EPA (European Press/Photo Agency) dans l'IHT (lundi 24 juillet 2006), en page 4, contextualise : « Hamas et Hezbollah : Le premier ministre de Palestine, Ismail Haniya du Hamas (photo), représente l'un des deux groupes terroristes qui font aussi partie de la politique mainstream ». Cela n'est pas faux - dans le gouvernement libanais, le Hezbollah, lui, est représenté - ce qui, toutefois, constitue un prisme. Prisme de la nomenclature des autorités états-uniennes et européennes qui désignent tel ou tel groupe ou État de «terroriste ». Par des reportages, correspondances, citations d'experts et commentaires des éditorialistesmaison ou de chroniqueurs invités à l'appui, l'IHT donne un éventail d'informations et de points de vue. Mais le prisme dominant reste « États-Unis/lsraël centré ». Pour expliquer le comportement du Hezbollah, le chroniqueur-maison, John Tierney, renvoie à un ouvrage sur I'histoire des codes d'honneur : «Israël continue à dire que son armée ne vise que les cibles militaires, mais le Hezbollah n'essaie même pas de faire semblant. Avec fierté, ses soldats tirent sur les civils. Ces terroristes se tiennent pour des hommes d'honneur, et malheureusement, ils le sont, d'après leur propres critères » (IHT, 26/07/06: 7). En outre, la présentation de l'attitude des autorités arabes souligne leurs craintes devant la popularité du Hezbollah, et questionne parfois la matrice des représentations courantes - la Syrie qui depuis longtemps affirme une politique d'accueil à l'égard de toutes les populations arabes, se montre soucieuse d'accueillir autant de réfugiés libanais que possible, mais redoute d'être débordée (IHT, 26/07/06:4)...

De cette lecture rapide, en diagonale, de trois titres-phares pendant les premiers dix jours de l'offensive d'Israël, il ressort que la vision dominante reflète un prisme « États-Unis/lsraël », la couverture et principal éditorial de The Economist du 22 juillet titre : « The accidental war » [La guerre par accident] : «Personne probablement n'en voulait et personne ne peut la gagner » (ibid. : I I). Le 24 juillet, The Times de Londres fulmine, lui, contre une émission du « débat de prestige » de la BBC, au cours duquel aucun défenseur d'Israël n'a été invité à s'exprimer (séquence « Commentaires », «The Thunderer 》, The Times, 24/07/06: 16). Dans la même séquence, un texte signé du rédacteur en chef du Jewish Chronicle s'intitule : « This time even Jews can agree ». Le Hezbollah a réalisé ce qui paraissait presque impossible : l'unité des quelques 300000 Juifs britanniques. Cette fois, même les Juifs en Grande-Bretagne peuvent se mettre d'accord (The Times, 24/07/06 : 17). 
On connait les dangers de choisir ainsi quelques textes. Mais le chercheur doit-il scruter autrement le discours média qu'il analyse, le contenu qu'il mesure, les représentations qu'il détecte? Certains répondraient positivement ; d'autres soutiendraient que le journal The Times faisant partie du groupe News Corp que dirige Rupert Murdoch, est tenu pour favorable à une vision Bush-Blair (pro-Israël) du monde et que pour analyser sa copie, point besoin d'aller chercher midi à quatorze heures...

Daniel Dayan affine avec davantage de subtilité la complexité des regards à porter sur la copie médiatique. Néanmoins, je crains que le terme « journalisé », avatar presque du journal télévisé, et qu'il préfère à « journalistique » (péjoratif) connaisse le même sort qu'il releva pour « Serial Killers », la formule d'Éric Fottorino du Monde, à propos de l'État israélien, (Dayan, 2005 : 199), certes, pour d'autres motifs. On rappellera qu'en anglais 《 journalese 》, terme repéré dès |882, et qui signifie la copie payée à la ligne, a eu la vie longue. Que Daniel Dayan campe plus du côté de la réception que moi, cela n'est pas la question. On se retrouve peut-être à propos de deux domaines, aux vocables nombreux, mais que l'on peut résumer par «l'interface espace média/espace public » et «le consensus/dissensus : ses courants dominants et contre-courants émergents ». Les courants contradictoires, à force de faire tourbillonner le « Mainstream », changent quelque peu le cours dominant...

Outre-Manche, il fut un temps où « la réaction » au contenu de l'offre média était d'écrire un courrier. « Letters to The Times », « Talk Back »; « Any Answers », ainsi se nommaient des rubriques ou émissions où un média dominant (The Times, BBC - télévision et radio) publicisait certaines des réactions suscitées par ce qu'il diffusait. Les points de vue relevés s'inscrivaient à l'intérieur des marges d'une expression de bon aloi, du désaccord poli et médié : gentlemen agree to disagree. Aujourd'hui, les sites en ligne des radiotélévisions, journaux et agences, croulent sous l'avalanche des réactions suscitées par leur copie ; les marges du dicible s'élargissent, la part des injures de même. Les échanges sur l'intranet et l'internet foisonnent ; la blogosphère grandit plus rapidement qu'à vue d'œil. Dans l'ouvrage précité de Joss Dray et Denis Sieffert (2002 : | | 3-1 | 7), les auteurs traitent à la fois des dérapages et du harcèlement des médias et des journalistes très en vue dans les années 2000-2002. Un ami de l'AFP m'a indiqué que sa maison se vit traitée d'« Agence France Palestine »... Reuters et AFP, à Londres, aux États-Unis, à Paris relevaient les « bombardements 》 de leurs sites par des internautes parfois agissant de manière orchestrée : on organise une campagne de clics (« hits ») pour critiquer l'écart entre telle photo - de l'angle de tir d'un soldat visant un civil - et la légende qui l'accompagne; on la scrute au point de déceler des intentions autres que celles que suggère le mot « viser ». Les diasporas se mobilisent et les mieux dotés en informatique tiennent le haut du pavé. Aux journalistes professionnels, tout cloués au pilori qu'ils soient, de tenir bon... Ils s'exposent métaphoriquement comme leur confrères 
s'exposent pour de vrai : une journaliste-photographe libanaise est tuée dès le 23 juillet 2006, douzième jour de la guerre, alors que Libanais et Israéliens dialoguent par blog interposé. La contestation de ces «pros»par le politique et par les détenteurs de tout pouvoir est chose ancienne. Celle exprimée par les communautés en ligne s'y ajoute : serait-ce comme dit l'américain une forme d'empowerment, de revendications contestataires, d'affirmation de soi en contestant pouvoirs et vecteurs-médias traditionnels, aussi « pluralistes » soient-ils ? Un « soi » où la recherche identitaire se conjugue avec l'expression d'un « nous autres » plus ou moins organisé, traversé de colères et friand de coups ponctuels?

\section{Conclusion}

Daniel Dayan est un chercheur engagé. L'engagement est une force : elle aide à déplacer les montagnes - les représentations que s'en font d'autres, tout au moins. La recherche engagée n'est pas celle des médias. « Le journaliste n'est a priori ni historien ni sociologue » (Dray, Sieffert, 2002 : 99). Aujourd'hui, les « info-comm'iens » scrutent comment la blogosphère s'ajoute à « l'en-lign'osphère », à la vidéosphère et à la graphosphère. Enrichissent-ils, dans le désordre, cet espace public que Daniel Dayan veut voir renforcé ? À court terme, on pourrait croire que cette démocratie électronique, participative - grille d'analyse que certains mettent en avant - n'est pas forcément la référence pertinente. Les intranets des entreprises d'une part, les blogs de tout un chacun, d'autre part, sont parfois des ersatz de la communication, soupapes ou canaux d'Ego et de Narcisse. À long terme, on n'en sait rien : le diplomate, lui, pourrait reprendre à ce sujet le discours « voix multiples, un seul monde $\gg .$. . Daniel Dayan, pour sa part, en braquant la focale sur les tenants et aboutissants « micro » nous convainc que la multiplicité des postures du chercheur n'atténue aucunement l'indignation du citoyen du monde. II s'enrage de pouvoir peu. Mais il agit quand même.

\section{Références}

Agence France Presse, 2004, Le manuel de l'agencier, Paris, AFP.

Chomsky N., Herman E. S., 1988, Manufacturing consent. The Political Economy of the Mass Media, New York, Pantheon Books.

Dayan D., dir., 2006, Le théâtre de la terreur. Terrorisme et télévision, Bruxelles, De Boeck Université/INA.

Dray D., Sieffert J., 2002, La guerre israélienne de l'information. Désinformation et fausse symétries dans le conflit israélo-palestinien, Paris, Éd. La Découverte.

Herman E. S., Chomsky N., 1988, Manufacturing Consent :The Political Economy of the Mass Media, New York, Pantheon. 
Lecture de Daniel dans la fosse aux lions

Palmer M., 2006, Dernières nouvelles d'Amérique : médias, pouvoirs et langages depuis les États-Unis (XVIII - XXle siècles), Paris, Éd. Amandier.

Philo G., Berry M., 2004, Bad news from Israel, London, Pluto.

Said E., 198I, Covering Islam : How the media and the experts determine how we see the rest of the world, London, R. K. Paul/Vintage/Random House, 1997. 